\title{
Direction of Gene Therapy and Virotherapy
}

\section{Masato Yamamoto*}

Division of Basic and Translational Research, Department of Surgery, University of Minnesota, Minneapolis, MN 55455, USA

Gene therapy is a group of therapies to treat diseases by expressing gene in the body. Originally, the concept was initiated for complementation of the defective gene for hereditary diseases (e.g. ADA-SCID "the bubble boy disease, but was soon expanded to acquired diseases which include a variety of benign and malignant diseases. In this sense, the gene therapy is now a broad concept representing therapeutics which treats hereditary and acquired diseases by introducing transgene into the target cells/organs.

Basically, clinical functionality of gene therapy modalities are determined by the gene transfer efficiency defined by the vector system as well as the level of gene expression/transduction required to achieve the clinical effect in the target diseases. From the view point of disease specific requirement of transduction level, these numbers are largely different depending on what disease we are talking about. For hemophilia, blood coagulation factor level even at a few percent of normal control makes significant clinical impact for avoidance of bleeding from micro injuries. On the other hand, cancer treatment may require virtually $100 \%$ transduction. From the stand point of vector efficiency, most of non-viral methods tend to show low efficiency although they are less toxic in most cases. Viral vectors taking advantage of viral life cycle as "naturally engineered gene transfer machinery" are very efficient, but have drawbacks as a pathogen. Virotherapy, alternatively called oncolytic viruses, is a kind gene therapy which utilizes replication competent virus for achieving oncolysis as a result of viral replication cycle and is expected to overcome the issue of transduction efficiency by permitting cancer specific viral replication and intratumoral spread. Thus, virotherapy shares the same challenge for clinical significance with other gene therapy modalities.

In 1995, Dr. Harold Varmus, Director, National Institutes of Health $(\mathrm{NIH})$, appointed an ad hoc committee to assess the status and promise of gene therapy and provide recommendations regarding future NIH-sponsored research in this area. So called Orkin-Motulsky Report [1] from this committee pointed out "Major difficulties at the basic level include shortcomings in all current gene transfer vectors and an inadequate understanding of the biological interaction of these vectors with the host." So, the gene therapists at that era started variety of researches along with this line, and even now efforts to improve gene therapy modalities still are trying to answer the suggestions in this report. To this end, lots of vector improvements were made and a variety of experiments for analyzing vector-host interaction has been performed in both hereditary and acquired diseases.

In the gene therapy in hereditary diseases, there are several breakthrough clinical outcomes published recently. ADA-SCID gene therapy with retrovirus vector achieved expected corrections, meaning adequacy of concept, while emergence of lymph proliferative disease due to insertional geno-toxicity due to older version of the vector, which mandates further improvement of the vector [2]. (Geno-toxicity observed in this study is often stressed but achievement of clinical correction is very important point of this study as well.) AAV-2 vectorbased gene therapy for RPE65-associated Leber's congenital amaurosis achieved good response [3]. More recently, a paper by Nathwani et al. [4] about gene therapy of hemophilia B with adenovirus-associated virus (AAV) vector was impressive. Infusion of a single dose of a serotype-8-pseudotyped, self-complementary AAV vector expressing a codon-optimized human factor IX(FIX) transgene (scAAV2/8-LP1hFIXco) in a peripheral vein achieved expression of FIX at 2 to $11 \%$ of normal levels, and four of the six discontinued FIX prophylaxis and remained free of spontaneous hemorrhage. This positive outcome is a result of logical vector design and right choice of target disease. AAV has been used for the gene delivery for hereditary diseases but there was an issue of time line for transgene expression. The single strand DNA virus takes weeks before the virus genome is converted to double strand leading to high level transgene expression. This issue was dealt with self-complementation although this modification reduces the limit of the size of the transgene. The pseudotyping of the virus with AAV8 capsids permits favorable distribution profile without sacrificing efficient expression system derived from AAV2. More importantly, the choice of the disease was just right. The avoidance of symptomatic bleedings require only tiny fraction of restoration compared to the normal level of factor IX. However, most diseases that achieved clinical effects were so far either the ones low level complementation can make clinical significance or strong selection/survival benefits exists in genetically corrected cells. In this sense, treatment of many hereditary enzyme deficiencies is still difficult since it requires high percentage of correction and the clear survival benefit of the corrected cells. In this meaning, in vivo selection of the transduced cells reported by Paulk et al. [5] is very interesting approach and may solve the issue of low correction rate. The most important take-home message is that logical vector design and rational clinical protocol are expected to enable clinically meaningful gene expression by gene therapy.

In acquired diseases, most frequent target of gene therapy is cancer because its lethality makes acceptable risk of the treatment higher. On the other hand, the challenge for clinical efficacy is higher. Since the target population of cancer gene therapy/virotherapy is currently the group of patients with unresectable diseases, most patients have metastases or locally advanced lesions. The treatment of metastatic lesions requires the therapeutic effect via systemic administration or the vector function which achieves therapeutic effect in the tumors other than the originally injected region. For the locally advanced

*Corresponding author: Masato Yamamoto, MD, PhD, Co-Director, Professor Division of Basic and Translational Research, Department of Surgery, University of Minnesota, MoosT 11-210, 515 Delaware St SE, Minneapolis, MN 55455, USA, Tel: 612-624-9131; E-mail: yamam016@umn.edu

Received November 20, 2012; Accepted November 23, 2012; Published November 26, 2012

Citation: Yamamoto M (2013) Direction of Gene Therapy and Virotherapy. J Cancer Sci Ther 5: e118. doi:10.4172/1948-5956.1000e118

Copyright: () 2013 Yamamoto M. This is an open-access article distributed under the terms of the Creative Commons Attribution License, which permits unrestricted use, distribution, and reproduction in any medium, provided the original author and source are credited. 
lesions, the transduction efficiency becomes the biggest issue since the residual untreated cancer cells in the tumor will lead to the quick relapse of the disease.

A variety of efforts has been made to enable systemic treatment of cancer. In the field of adenoviral vectors where our group is working, capsid modification is a way to change the behavior after intravenous delivery. Recent focus of adenovirus capsid modification is hexon protein. Various alternations in hyper-variable regions of adenovirus hexon protein reduce the sequestration of the virus to reticuloendothelial system (i.e. liver and spleen) via reduction of reaction with the coagulation factor X [6], which is related to the Ad induced innate immunity also [7]. In this sense, hexon modification makes a lot of sense in the context of extension of blood circulation half-life as well as reduction of toxicity.

On the other hand, selective transduction of target cancer cells without reducing the efficiency upon systemic delivery has been a big challenge for all gene delivery systems. Wind-type adenoviruses bind to the target cells via the binding of the fiber-knob region to the receptors on the surface of the target cells. Thus, it is logical to incorporate the selective ligand into the fiber knob region, but vast majority of the efforts fail because of rigidity of the fiber structure. After most modifications, the adenovirus cannot be assembled efficiently or loses the binding affinity even in case they are successfully assembled. Thus, identification of functional ligand is theoretically possible but is actually like "finding a needle in a haystack". Recently, we developed a novel technology enables high efficiency Ad vector production and applied it for adenovirus targeting motif library and its high throughput screening. The screening of the Ad library $\left(5 \times 10^{9}\right.$ diversity) with target moiety expressing cells led to the identification of novel targeting motif permitting systemic targeting to the target-expressing tumor [8]. It is hoped that such advance in vector engineering enables treatment of cancers via systemic administration.

In the context of the therapeutic effect in uninjected lesions, immunotherapy approach is very interesting and promising. In genetic vaccine clinical trial with allogeneic GM-CSF secreting tumor immunotherapy $\left(\mathrm{GVAX}^{\star}\right)$, some pancreatic cancer patients showed delayed-type hypersensitivity and the target was mesothelin [9]. Another interesting approach in pancreatic cancer is interferon expressing adenovirus vector reported by Ohahsi et al. [10]. Adenovirus injection into one side of the tumor regressed the tumor on the counter side, and this effect was suppressed by NK cell depletion. This provides evidence that gene therapy with immunostimulatory molecule can provide therapeutic effect on the tumors which are not directly treated.

The transduction efficiency issue is a major issue because required efficiency for suppression of tumor growth is high. This is the reason that the approach producing bystander effect (e.g. HSV-TK with ganciclovir) has been preferred for gene therapy compared to the molecules whose effect is limited to the transduced cells. One interesting concept which has potential to be used for this purpose is radionuclides. A variety of radionuclides have been used for radiotherapy of cancers (e.g. ${ }^{131}$ I). If gene therapy approach can achieve selective accumulation of radionuclides to the level required for therapeutic effect, the clinical impact would be big. Dwyer et al. [11] reported such approach in prostate cancer models.

Another approach for transduction efficiency is to permit the injected virus to replicate in the tumor locale. The produced progeny virus infects the surrounding cells and continues to spread. If the virus is designed to replicate selectively in cancer cells, the virus will produce ultimate bystander effect by its spread and cytocidal effect specifically in cancer cells. Many viruses have been studied for this direction. Adenoviruses have been studied a lot by many groups including ours. Major benefits of Ad are well understood virology and good size for genetic manipulation. Thus, Ad is one of the best choices for detail virus engineering. The genetically modified first oncolytic adenovirus, $\mathrm{H} 101$, is the first virus approved by a regulatory agency in the world [12]. Many more advanced generation oncolytic adenoviruses are being developed and tested. More recently, oncolytic viruses based on herpes simplex virus (OncoVEX GM-CSF ${ }^{13}$ ) and vaccinia ${ }^{14}$ entered phase III clinical trial with high anticipation of clinical impact. In this sense, oncolytic virus is very attractive field for cancer gene-/viro-therapy, and a lot of vector engineering is going on for the development of next generation oncolytic viruses $[13,14]$.

As described above, gene therapy researchers are improving the vectors and treatment regimen along with Orkin-Motulsky Report. However, this does not mean there isn't much advance. The researcher in the field of gene-/viro-therapy have made significant advance both technologically and clinically. Simply, the report grasps the core feature and issue of gene therapy, and thus many issues mentioned in the report will continue to be the guideline for the improvement of gene therapy and its related technologies. Logical vector design and rational clinical protocol will lead to clinical impacts of gene therapy.

\section{References}

1. Orkin SH, Motulsky AG (1995) Report and recommendation of the panel to assess the $\mathrm{NIH}$ invesment in research on gene therapy.

2. Blaese RM, Culver KW, Miller AD, Carter CS, Fleisher T, et al. (1995) lymphocyte-directed gene therapy for ADA- SCID: initial trial results after 4 years. Science 270: $475-480$

3. Maguire AM, Simonelli F, Pierce EA, Pugh EN Jr, Mingozzi F, et al. (2008) Safety and efficacy of gene transfer for Leber's congenital amaurosis. N Engl J Med 358: 2240-2248.

4. Nathwani AC, Tuddenham EG, Rangarajan S, Rosales C, Mclntosh J, et al. (2011) Adenovirus-associated virus vector-mediated gene transfer in hemophilia B. N Engl J Med 365: 2357-2365.

5. Paulk NK, Wursthorn K, Haft A, Pelz C, Clarke G, et al. (2012) In Vivo Selection of Transplanted Hepatocytes by Pharmacological Inhibition of Fumarylacetoacetate Hydrolase in Wild-type Mice. Mol Ther 20: 1981-1987.

6. Alba R, Bradshaw AC, Parker AL, Bhella D, Waddington SN, et al. (2009) Identification of coagulation factor $(\mathrm{F}) \mathrm{X}$ binding sites on the adenovirus serotype 5 hexon: effect of mutagenesis on FX interactions and gene transfer. Blood 114: 965-971.

7. Doronin K, Flatt JW, Di Paolo NC, Khare R, Kalyuzhniy O, et al. (2012) Coagulation factor $X$ activates innate immunity to human species $C$ adenovirus. Science 338: 795-798.

8. Miura Y, Yamasaki S, Davydova J, Brown E, Aoki K, et al. (2012) Infectivityselective Oncolytic Adenovirus Developed by High-throughput Screening of Adenovirus-formatted Library. Mol Ther

9. Thomas AM, Santarsiero LM, Lutz ER, Armstrong TD, Chen YC, et al. (2004) Mesothelin-specific CD8(+) T cell responses provide evidence of in vivo crosspriming by antigen-presenting cells in vaccinated pancreatic cancer patients. $J$ Exp Med 200: 297-306.

10. Ohashi M, Yoshida K, Kushida M, Miura Y, Ohnami S, et al. (2005) Adenovirusmediated interferon alpha gene transfer induces regional direct cytotoxicity and possible systemic immunity against pancreatic cancer. Br J Cancer 93: 441449 
11. Dwyer RM, Bergert ER, O'connor MK, Gendler SJ, Morris JC (2005) In vivo radioiodide imaging and treatment of breast cancer xenografts after MUC1driven expression of the sodium iodide symporter. Clin Cancer Res 11: 14831489.

12. Frew SE, Sammut SM, Shore AF, Ramjist JK, Al-Bader S, et al. (2008) Chinese health biotech and the billion-patient market. Nat Biotechnol 26: 37-53.

13. Kaufman HL, Bines SD (2010) OPTIM trial: a Phase III trial of an oncolytic herpes virus encoding GM-CSF for unresectable stage III or IV melanoma. Future Oncol 6: 941-949.

14. Parato KA, Breitbach CJ, Le Boeuf F, Wang J, Storbeck C, et al. (2012) The oncolytic poxvirus JX-594 selectively replicates in and destroys cancer cells driven by genetic pathways commonly activated in cancers. Mol Ther 20: 749758 